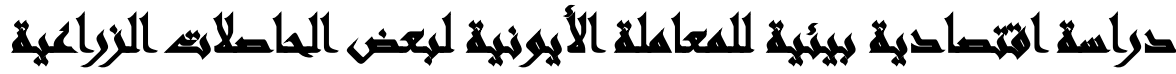

\section{[؛]}

علا صلاح عبد المجيد(')- محمد سيد شحاتة(؟)- عصام عبد الرحمن الجميل(')

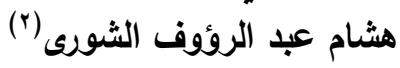

( ) هيئة الطاقة الذرية ب) كلية الزراعة، جامعة عين شمس الروف

\section{المستخلص:}

نظرا للمشاكل البيئية والصحية لإستخدام الكيماويات فى حفظ الأغذية فقد أعدت هذه

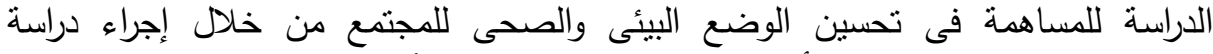

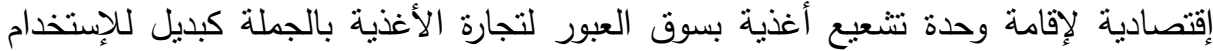

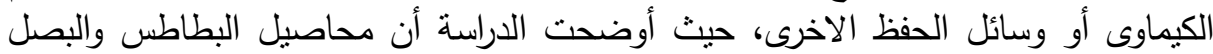

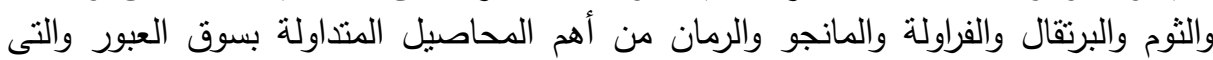

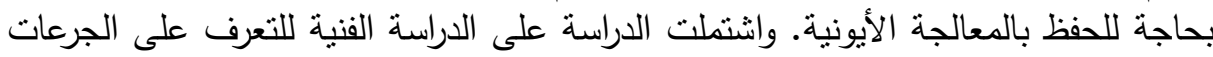

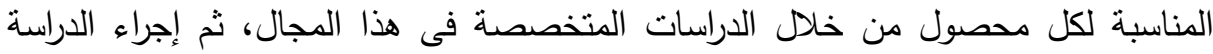

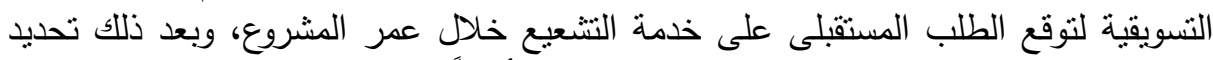
نموذج الوحدة المناسبة من المعجلات الإلكترونية، وأخيراً إجراء التحليل المالى لكلى لكل مقترح

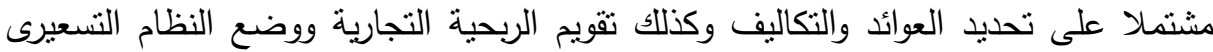
وكذلك تحديد العوائد القومية بما بساهم فى تحسين القدرات التسويقية لهذه الحاصلية العاتلات الزراعية

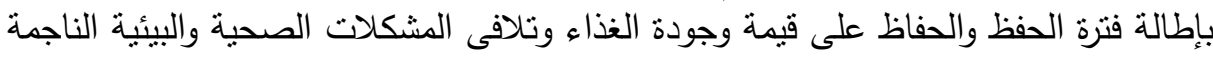

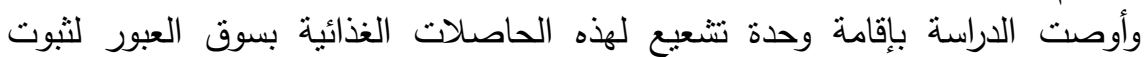

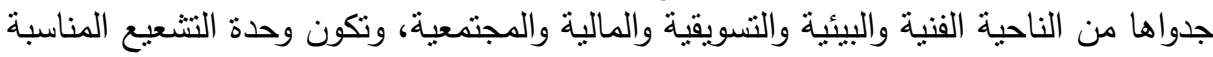

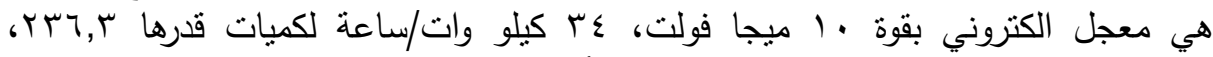


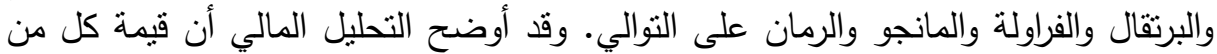

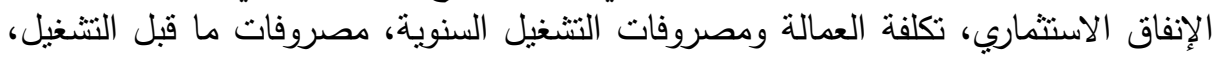

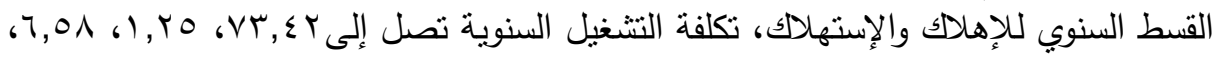

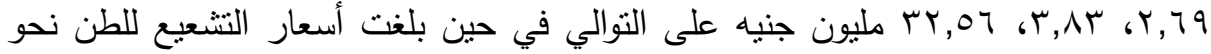

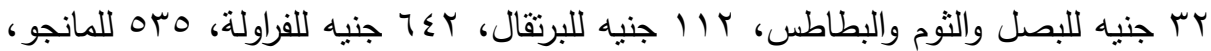

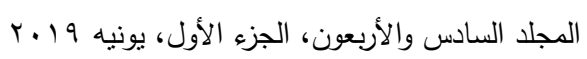




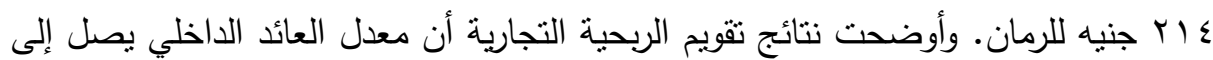

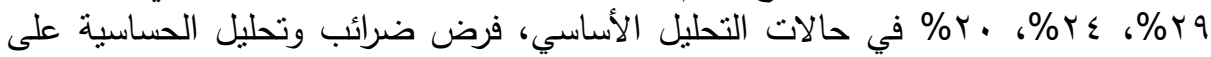

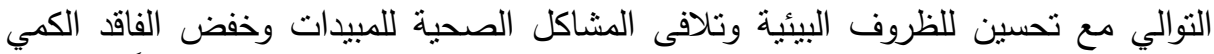

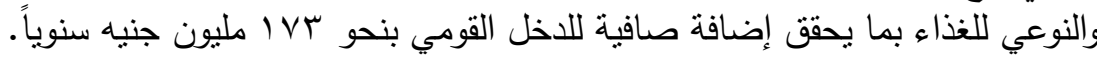

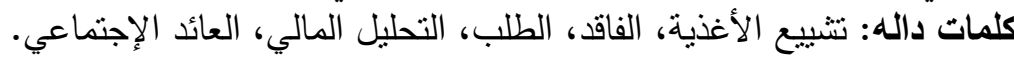

\section{axis}

تعنبر تكنولوجيا التشعيع الغذائى واحدة من أهم التقنيات الحديثة والتى تتلافى مشاكل

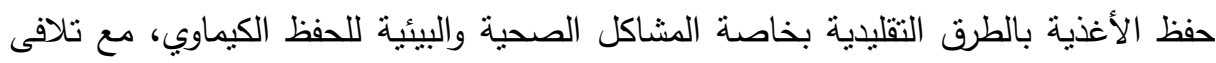
مشاكل الفاقد الكمى والنوعى بعد الحصاد، والحفاظ على الصورة الطازجة للغذاء وقيمتة وجودنة وهى منتباة على المستوى الدولى من كل من منظمة الصحة العالمية، ومنظمة

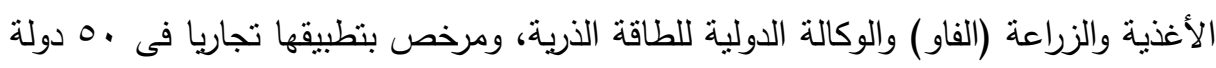

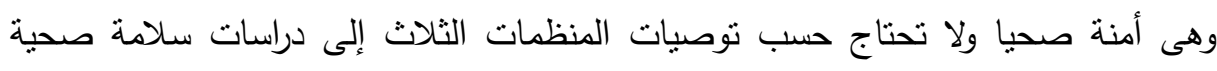

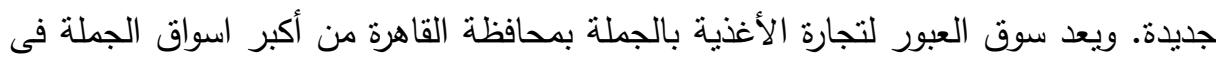

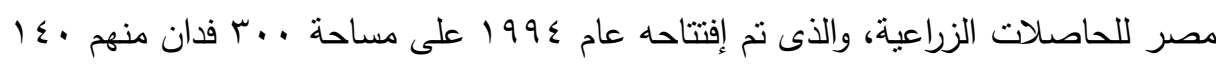

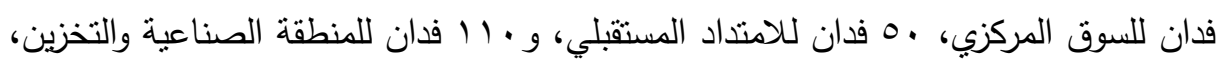

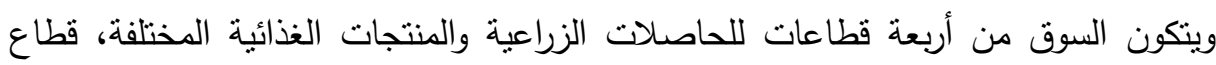
الخضر، الفاكهة، الموز، السمك والدواجن. ويصل منوسط الكميات المتداولة بسوق العبور

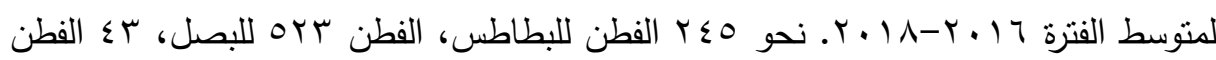

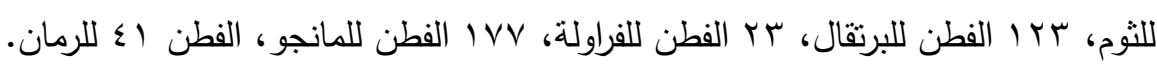

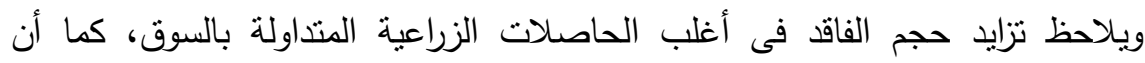
الطرق التقليدية المستخدمة للحفظ داخل السوق منل الكيماويات لها مضار صحية وبيئية وتسويقية، ووسائل التبريد المستخدمة غير قادرة على استيعاب الكميات المتداولة بالسوق وفاعليتها ليست جيدة فى حفظ الحاصلات الزراعية. 


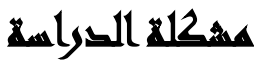

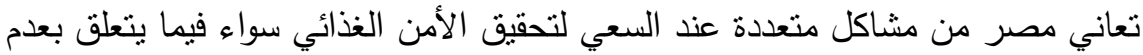

كفاية الإنتاج المحلي لاحتياجات الأفراد، أو من مثاكل الفاقد الكمي والنوعي للحاصلات الألات

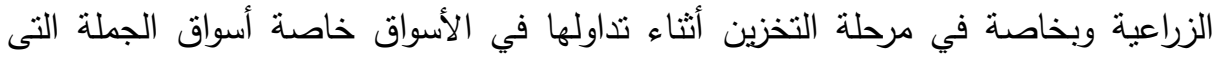

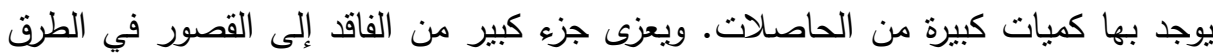
التقليدية المنبعة في الحفظ، بالإضافة إلى المثاكل البيئية والصحية والاقتصادية المرتبطة باستخدام هذه الطرق.

\section{أهمية الصراسمة}

تتمنل أهمية الدراسة بالمساعدة في خفض الفجوة الغذائية في مصر من خلال المساهمة في حل مشاكل الفاقد الكمي والنوعي للغذاء في مرحلة ما بعد الحصاد من خلال تقديم الروئة الاقتصادية لإمكانية استخدام تكنولوجيا التشعيع الغذائي، باعتبارها أحدث التقنيات المطروحة لتجنب المشاكل الاقتصادية والبيئية والفنية والصحية للطرق التقليدية وذلك في سوق العبور لتجارة الأغذية بالجملة بمحافظة القاهرة والذى ينم خلاله تداول كميات كبيرة من الحاصلات

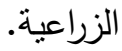

\section{منهمجية القراسة}

تتبع هذه الدراسة منهجية البنك الدولي في إجراء دراسات الجدوى، حيث تهتم بالنواحي المتعلقة بجدوى إقامة وحدات نتعيع الحاصلات الزراعية من حيث الدراسة الفنية والهندسية والتسويقية ودراسة التكاليف والعوائد ووضع النظام التسعيري المناسب وتقويم الربحية التجارية وكذلك دراسة العوائد القومية لنطبيق تكنولوجيا التشعيع الغذائي في مصر . 


\section{مساقر الميهانهايت}

بيانات ثانوية: سوق العبور لتجارة الأغذية بالجملة بمحافظة القاهرة، هيئة الطاقة الذرية

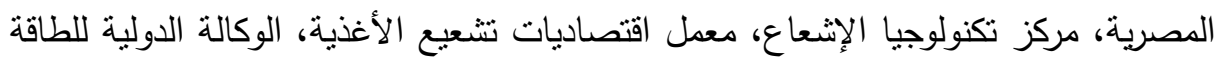

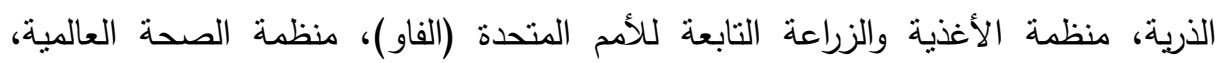
المنظمة العربية للتتمية الزراعية، وزارة الزراعة المصرية، الجهاز المركزي للتعبئة العامة والإحصاء، شبكة المعلومات الدولية.

\section{السرواسمة اللحسوريليد}

تهدف الدراسة التسويقية إلى تقديم خطوط إرشادية حول الإمكانيات المستقبلية المتوقعة للطلب على خدمات تشعيع كل من محاصيل البطاطس، البصل، الثوم، البرتقال، الفراولة،

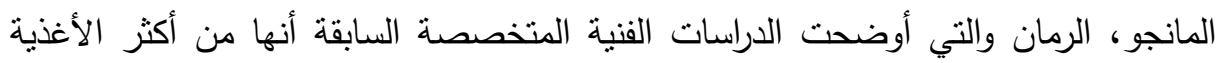
حاجة وصلاحية للحفظ بالتشعيع باسواق الجملة. ويبين جدول (1) تطور الكميات المتداولة

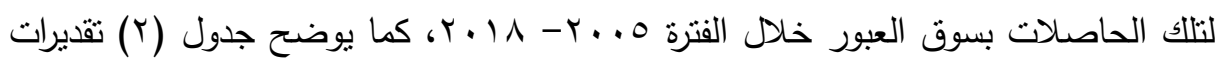
الاتجاة الزمنى الخطيه لتلك الحاصلات. ويتبين الاتجاه المتزايد للكميات الواردة من محاصيل

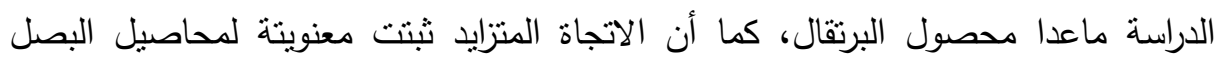

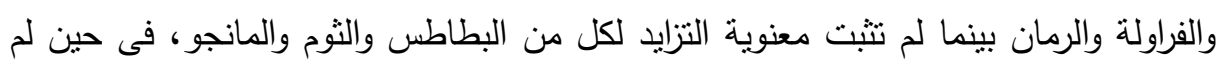
تثبت معنوية تتاقص الكميات الواردة للسوق من البرتقال. 
مجلة العلوم البيئية

معهد الدراسات والبحوث البيئية - جامعة عين شمس لئه

جدول (1): الكميات المتداولة من بعض الحاصلات الزراعية الغذائية بسوق العبور لتجارة

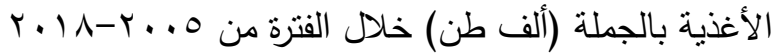

\begin{tabular}{|c|c|c|c|c|c|c|c|}
\hline الرمان & المانجو & الفراولة & البرتقال & الثوم & البصل & البطاطس & السنة \\
\hline $1 \pi$, & Y $70, \Lambda$ & $\Lambda, \xi$ & $r \mid r, \varepsilon$ & $1 \leqslant, Y$ & $1 \pi \cdot, \varepsilon$ & $1 \leqslant 9, \varepsilon$ & r... \\
\hline $17,$. & $\sum 9$ & $\vee, \Lambda$ & $\Lambda \wedge, \wedge$ & $19, \pi$ & $|r|, \varepsilon$ & $\Pi 1 Y, 1$ & $T \ldots T$ \\
\hline $17, V$ & $v 9,1$ & $r, 0$ & $T Y, r$ & $r q, v$ & $17 \cdot, r$ & $10 \leqslant, 7$ & $T \ldots V$ \\
\hline$Y 7, V$ & $\Lambda \vee, \Lambda$ & $0, \Lambda$ & $9 \xi, \varepsilon$ & $71, \Lambda$ & $\varepsilon Y \cdot, \xi$ & $r q \varepsilon, v$ & $r \ldots h$ \\
\hline 17,0 & rqA & $Y, 7$ & YTr,A & $Y 7,7$ & $T V \cdot, \varepsilon$ & $r \cdots, r$ & $r \ldots q$ \\
\hline $1 \pi, 0$ & $1 \cdots, V$ & $\Lambda, 9$ & $T \cdot \varepsilon, T$ & $0 ., 1$ & $\varepsilon Y \leqslant, Y$ & $\varepsilon \cdot \wedge, \cdot$ & $T .1$. \\
\hline$r \mu, \cdot$ & $177, V$ & $\overline{V, 1}$ & $r 1 \cdot, q$ & $\| T_{,}$ & $0 \wedge 9, \mathrm{~V}$ & $0 . \mu$ & $r .11$ \\
\hline$r \cdot, r$ & $r r \Lambda, 1$ & $11, \varepsilon$ & 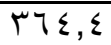 & 177,0 & $T Y \Lambda, V$ & 000,7 & $T . I T$ \\
\hline$\varepsilon r, 1$ & YTV, & YI, & $r r \cdot, 0$ & ITT,. & $09 V, Y$ & $0 \leqslant \varepsilon, V$ & $T .1 T$ \\
\hline $00, \mu$ & Y70,9 & $T V, \cdot$ & $T / \cdot, l$ & IYY,Y & $0 \cdot 1,9$ & $\xi O V, \Lambda$ & $T \cdot I \varepsilon$ \\
\hline$T \leqslant, Y$ & $T \leqslant \wedge, V$ & YO, r & TYT & 00,1 & $r \mid r, q$ & $\Gamma \backslash V, 1$ & $T .10$ \\
\hline r4 & 101,0 & $T \leqslant, 0$ & $1 \Gamma 0,0$ & $\Lambda, r$ & $9 \vee \mu, r$ & $1 \cdot Y, 1$ & $r .17$ \\
\hline$r 9,1$ & 194,1 & $Y Y, T$ & $11 \pi, 9$ & $0\}, \xi$ & $\Gamma \cdot \varepsilon, \Lambda$ & $r r \varepsilon, r$ & $T . I V$ \\
\hline$\varepsilon 7, V$ & $1 \wedge 0, \varepsilon$ & $\overline{Y Y}$ & $11 \wedge, 7$ & $T V, r$ & rq1,7 & rq9 & $r .1 A$ \\
\hline
\end{tabular}

المصدر: سوق العبور لتجارة الأغذية بالجملة

جلول (Y): تقديرات الاتجاه الزمني لكمية الحاصلات الزراعية المحتمل تشعيعها والواردة

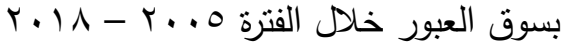

\begin{tabular}{|c|c|c|c|c|c|c|}
\hline PF & $\mathbf{F}$ & R2 & TB.C & التقايرات & الرياضية" * & لمحصول \\
\hline., ro $_{0}$ &., 97 & $\cdot, \cdot V$ & $\cdot, 91$ & $Y=254.7+10.39 x$ & الخطية & 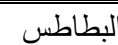 \\
\hline$\cdot, \cdot, 1$ & $r, v$ & $\cdot, r \leqslant$ & 1,94 & $\operatorname{Ln} Y=182.6+0.09 X$ & الاسية & البصل \\
\hline 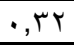 & $1, .9$ & $\cdot, \cdot 1$ & $1, .0$ & $Y=41.26+3.37 x$ & الخطية - م الخية & 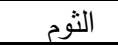 \\
\hline$\cdot, \mathrm{AV}$ & $\cdot, \cdot r$ & $\cdot, \ldots r$ &., 17 & $Y=200.5-1.03 x$ & الخطية - م الخية & البرتقال \\
\hline 7,1 & $r 0, r$ & $\cdot, \mathrm{V}_{0}$ & $0,9 \varepsilon$ & $Y=0.61+1.71 x$ & الخطية - م الخية & الفراولة - م الفي \\
\hline$\cdot, \mathrm{YA}$ & $1, Y_{1}$ &., 1 & $1,1 \pi$ & $Y=144.9+6.27 x$ & الخطية - م الخية & المانجو \\
\hline$\cdot, \cdots 1$ & IV,T & $\cdot, \pi$ & $\varepsilon, r$. & $Y=9.8+2.5 x$ & الخطية & الرمان \\
\hline
\end{tabular}

• تم التقدير باستخدام الصور الرياضية المختلفة والتقديرات لافضل النتائج.

Y Y الكمية المتداولة من المحصول بالألف طن، X X : : الزمن

(المصدر: حسبت من جدول (1)

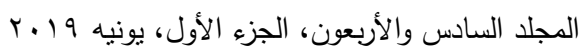


التقدير المستقبلي المتوقع للحاصلات الزراعية المحتمل تشعيعها بسوق العبور : يعتمد تقدير الكميات المتوقع تتعيعها في المستقبل على التتبؤ بالكميات المتوقع ورودها بسوق العبور

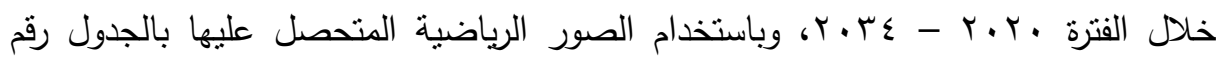
(ץ)، يوضح جدول (r) الكميات المتوقع ورودها لسوق العبور من الحاصلات الزراعية البصل والفراولة والرمان، بينما يكون التوقع بباقى المحاصيل نظراً لان تقديرات الاتجاة الزمنى لكي

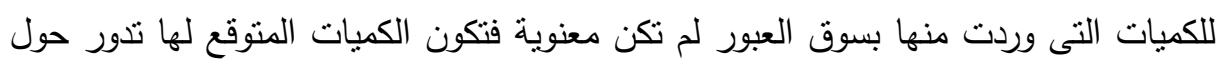
متوسطها الحسابى لفترة الدراسة.

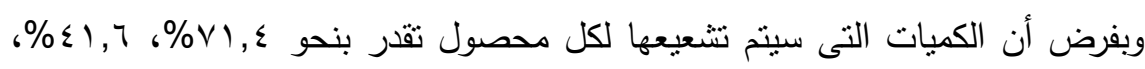

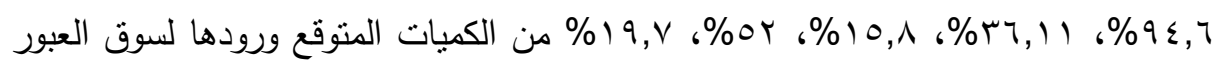

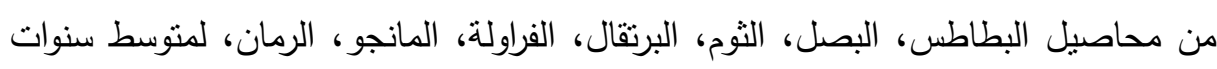

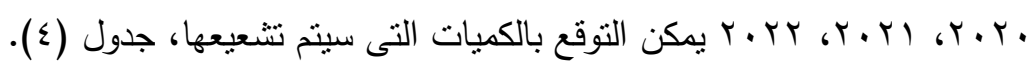

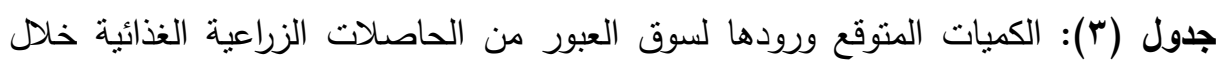

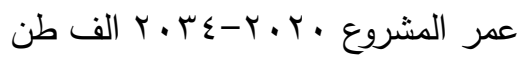

\begin{tabular}{|c|c|c|c|c|c|c|c|}
\hline الرمان & المانجو & الفراولة & البرتقال & الثَّوم & البصل & البطاطس & السنـة \\
\hline$\varepsilon 9,1$ & $I V A$ & $T V, 9 V$ & 194 & $7 V$ & $7 \leqslant 1,0$ & Tा & $T_{T \cdot Y \cdot}$ \\
\hline$O Y, r$ & $I V A$ & $Y \wedge, 7 \Lambda$ & 194 & $7 V$ & $77 \Lambda, \Lambda$ & וT & Y.YI \\
\hline $0 \xi, \Lambda$ & $I V \wedge$ & $\Gamma, 199$ & 194 & $7 V$ & 797,1 & TH & $\overline{Y \cdot Y Y}$ \\
\hline$O V, r$ & 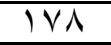 & $r \mu, 1$ & 194 & $7 V$ & VYT, & TH & $\overline{Y \cdot Y T}$ \\
\hline 09,1 & $T \vee \wedge$ & $\Gamma \varepsilon, \lambda 1$ & 194 & $7 V$ & $V 0 \cdot, V$ & וTr & $r \cdot Y \xi$ \\
\hline$T Y, r$ & $T \vee A$ & MY,OY & 194 & $7 V$ & $V \vee A$ & ו וT" & Y.YO \\
\hline $7 \varepsilon, 1$ & 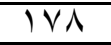 & $\Gamma \Lambda, Y \mu$ & 194 & $7 V$ & $\Lambda \cdot 0, \Gamma$ & TT & Y.YY \\
\hline $7 V, r$ & 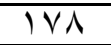 & $\Gamma q, 9 \varepsilon$ & 194 & $7 V$ & ATr,T & וT & $\overline{Y \cdot Y V}$ \\
\hline 79,1 & $T \vee \Lambda$ & $\leqslant 1,70$ & 194 & $7 V$ & 109,9 & וT & Y.YA \\
\hline$V Y, r$ & $T \vee A$ & $\varepsilon \Gamma, \Gamma\rceil$ & 194 & $7 V$ & $\Lambda \wedge \vee, r$ & וT & r.rq \\
\hline$V \varepsilon, \lambda$ & $T \vee \wedge$ & $\varepsilon 0, \cdot V$ & 194 & $7 V$ & $91 \leqslant, 0$ & וT & r.r. \\
\hline$V V, r$ & $I V A$ & $\varepsilon 7, \vee \wedge$ & 194 & $7 V$ & $9 \leq 1,1$ & THI & $r \cdot r)$ \\
\hline$\overline{V 9,1}$ & $T \vee \lambda$ & $\varepsilon \wedge, \varepsilon q$ & 194 & $7 V$ & 979,1 & TT & $\overline{T \cdot T Y}$ \\
\hline$\Lambda Y, r$ & $T \vee \wedge$ & $0 ., Y$ & 194 & $7 \mathrm{~V}$ & 997,5 & TT & $T \cdot T T$ \\
\hline$\Lambda \varepsilon, \Lambda$ & $T \vee A$ & 01,91 & 194 & $7 V$ & $1, Y T, V$ & וT & $T \cdot T \varepsilon$ \\
\hline $1 \ldots 9,0$ & YTV. & $09 \wedge, 1$ & TA90 & 1.00 & $T r \leqslant \wedge 9$ & $\sum 970$ & الإجمالى \\
\hline $7 V, \Gamma$ & $T \vee \lambda$ & $\Gamma q, \wedge V$ & 194 & $7 V$ & ATr, & Tा & المتوسط \\
\hline
\end{tabular}

المصدر: جداول(1)، (r) (ب) (1) 
جدول (§): الطلب المستقبلى المتوقع على إستخدام المعاملة الأيونية فى حفظ بعض

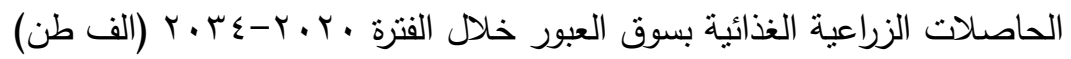

\begin{tabular}{|c|c|c|c|c|c|c|c|}
\hline الرمان & المانجو & الفراولة & البرتقال & اللثّوم & البصل & البطاطس & السنة \\
\hline $9, \wedge$ & $9 Y, 0$ & $\varepsilon, \xi$ & $79, V$ & $7 \varepsilon, 7$ & $\overline{r T r, r}$ & $\overline{r r q, r}$ & T.Y. \\
\hline $1 \cdot, r$ & $9 Y, 0$ & $\varepsilon, V$ & $79, V$ & $7 \varepsilon, 7$ & TVA & YT & $T \cdot Y{ }^{\prime}$ \\
\hline $1 \cdot, 9$ & $9 Y, 0$ & 0 & $79, V$ & $7 \varepsilon, 7$ & $r q \Psi, r$ & $r \Psi \bar{Y}, r$ & $T \cdot Y Y$ \\
\hline 11,0 & 94,0 & $0, r$ & $79, V$ & $7 \varepsilon, 7$ & $r \cdot \lambda$ & Trq, r & T.YT \\
\hline$T$ & 94,0 & 0,0 & $79, V$ & $7 \varepsilon, 7$ & TRT,Y & Trq, r & $r \cdot Y \varepsilon$ \\
\hline$T, T$ & 94,0 & $0, V$ & $79, V$ & $7 \leqslant, 7$ & או Tr & $T r q, r$ & Y.ro \\
\hline$T r, r$ & $9 Y, 0$ & 7 & $79, V$ & $7 \varepsilon, 7$ & Tos & $\Gamma M 7, r$ & r.r. \\
\hline$T r, V$ & $9 Y, 0$ & $7, r$ & $79, V$ & $7 \varepsilon, 7$ & rרג & $r r q, r$ & $T \cdot Y V$ \\
\hline$T \leqslant, r$ & 94,0 & 7,0 & $79, V$ & $7 \varepsilon, 7$ & rیr & $r \Psi \pi, r$ & $T \cdot Y \wedge$ \\
\hline $1 \varepsilon, 0$ & $9 Y, 0$ & 7,1 & $79, V$ & $7 \varepsilon, 7$ & $r q \Lambda, 1$ & 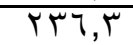 & $r \cdot r_{q}$ \\
\hline $10, \varepsilon$ & 94,0 & $V, 1$ & $79, V$ & $7 \varepsilon, 7$ & हाน, & $T r q, r$ & $r . r$. \\
\hline 17 & 94,0 & $\overline{V, r}$ & $79, V$ & $7 \varepsilon, 7$ & $\varepsilon Y \wedge, 1$ & $T \mu q, r$ & $T \cdot T$ \\
\hline 17,7 & 94,0 & $V, 7$ & $79, V$ & $7 \varepsilon, 7$ & $\varepsilon \varepsilon r, 1$ & Trq,r & T.TY \\
\hline $\mid V, 1$ & 94,0 & $V, 9$ & $79, V$ & $7 \leqslant, 7$ & $\leqslant 0 \wedge, 1$ & $r \mu q, r$ & 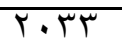 \\
\hline$I V, V$ & $9 Y, 0$ & $\lambda, 1$ & $79, V$ & $7 \varepsilon, 7$ & $\varepsilon V \Psi, 1$ & YT,$\Gamma$ & $T \cdot T \varepsilon$ \\
\hline$T \cdot 0, T$ & $T \Gamma \wedge V, 0$ & $9 \xi, 1$ & $1 \cdot \leqslant 0,0$ & 979 & $00 Y Y, Y$ & $r 0 \leqslant \leqslant, 0$ & لإجمالي \\
\hline$I T, V$ & $9 Y, 0$ & $7, r$ & $79, V$ & $7 \varepsilon, 7$ & $r \neg \Lambda, 1$ & $r \mu q, r$ & الّتوسط \\
\hline
\end{tabular}

المصدر: حسبت باستخدام جدولي (r، ()

\section{السراسمة الفنهية}

يمكن التشعيع الأيوني للحاصلات الغذائية باستخدام نوعان من التكنولوجيا وهي التشعيع

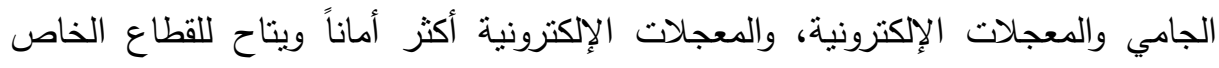

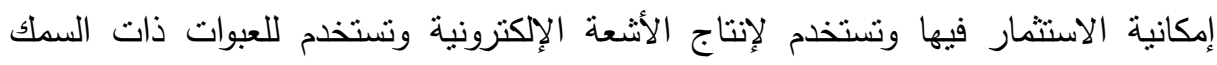

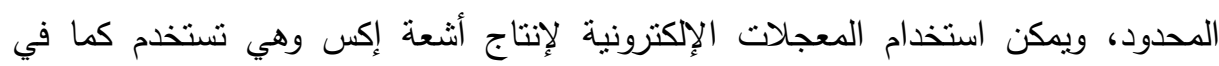

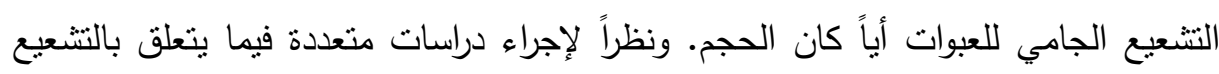
الجامي يقتصر البحث على دراسة المعجلات الإكترونية. 
النموذج الأمثل لوحدة التشعيع: يهنم هذا الجزء بتحديد وحدة التشعيع المناسبة لحفظ

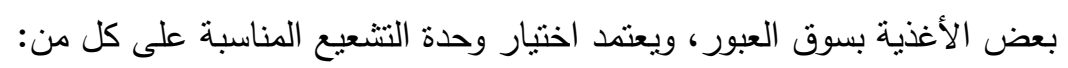

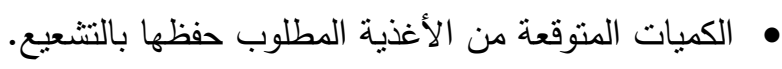
• الجرعة التشعيعية لكل محصول.

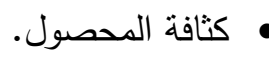

نظرا لاختلاف الجرعات التشعيعية لكل محصول، فان كل محصول سوف يحصل على

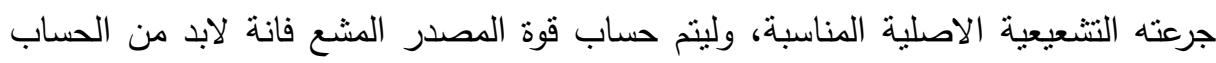
بناءا على جرعة تشعيعية موحدة (حسابياً) وهى ( لك.جراى ومن ثم فانه يتم تحديد الكمية

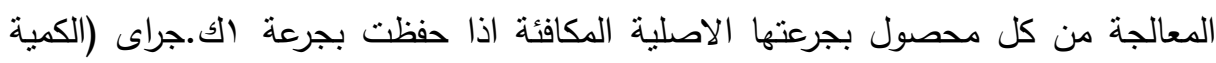
المعادلة)، وهو ما تم حسابة فى جدول (0) بإستخدام المعادلة الآتية:

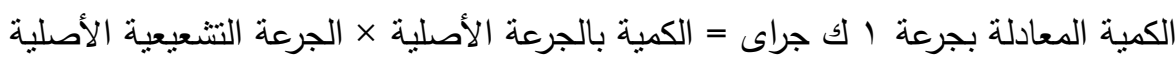
جدول (•): الكميات المعادلة من المحاصيل المراد حفظها بالتتعيع بسوق العبور (ألف طن)

\begin{tabular}{|c|c|c|c|}
\hline الكميلة المعادلة & الكمية بالجرعة الأصلية(1) & الجرعة التشعيعية المناسبة & المحصول \\
\hline$\Gamma_{0, \Sigma}$ & $\overline{r r q, r}$ &., 10 & البطاطس \\
\hline$\Sigma 1, V \Psi$ & $T \vee \Lambda, Y$ &., 10 & البصل \\
\hline 9,79 & $7 \varepsilon, 7$ &., 10 & ألنّم \\
\hline$r \leqslant, q$ & $79, V$ & $\cdot, 0$ & البرتقال \\
\hline $1 \varepsilon, 1$ & $\varepsilon, V$ & $r$ & الفراولة \\
\hline$r M, 1$ & $9 r, 0$ & $\cdot$, YO $_{0}$ & المانجو \\
\hline $1 \cdot, r$ & $1 \cdot, r$ & $1, \cdot$ & الرمان \\
\hline $179, \pi$ & \multicolumn{3}{|c|}{ الإجمالي } \\
\hline
\end{tabular}

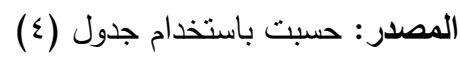

تحسب قوة المعجل الإكتروني بالكيلو وات وفقا للمعادلة الآتية:

$$
\frac{\tau \times(\omega \div 5)}{\varepsilon \times r q}=0
$$

حيث ق: قوة المعجل الإككترونى بالكيلووات ك: الكمية المطلوب تشعيعها بالكيلو جرام باليكات 


$$
\begin{aligned}
& \text { س: عدد ساعات التتغيل السنوية ( . .7 ساعة/سنة) }
\end{aligned}
$$

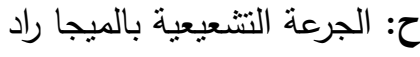

$$
\begin{aligned}
& \text { ع: كفاءة التشعيع : جرعه }
\end{aligned}
$$

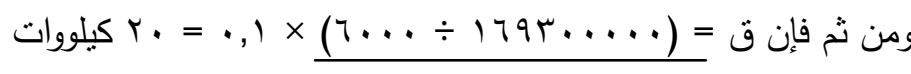

$$
\begin{aligned}
& \cdot, \varepsilon 1 \times 47 .
\end{aligned}
$$

ونظراً لإستخدام أشعة × فى حالة العبوات الكبيرة، وأن المعجل يعمل عند أثنعة × بنحو

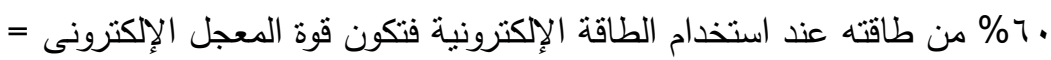

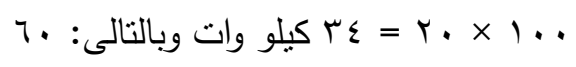

فإن مواصفات وحدة التشعيع من المعجلات الا لكترونية هى: معجل الكترونى ـ ( ميجافولت،

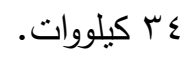

\section{اهنمراغايت السوالمة}

نوع وحدة التشعيع: معجل إلكتروني قوته = ع ك كيلووات، • ميجافولت.

عدد ساعات التشغيل: . . . 7 ساعة/سنة.

الوحدة النقدية المستخدمة: الجنية المصري.

البيانات السعرية: معمل اقتصاديات تشعيع الأغذية - هيئة الطاقة الذرية.

معايير تقويم الربحية التجارية المستخدمة: معدل العائد الداخلي، فترة الاسترداد ويتم

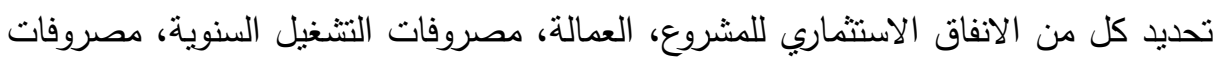
التأسيس، القسط السنوي لإهلاك واستهلاك الأصول الرأسمالية للمشروع. وكذلك وضع الإنع النظام التسعيري لوحدة التشعيع، ثم إعداد جداول التدفق النقدي للمشروع لتحديد عائد الربحية التجارية بالإضافة إلى تحليل الحساسية للمشروع مع دراسة للعوائد القومية. 


\section{أولاً: التدفقات الخارجة لوحدة المعجل الاكترونى بسوق العبور:}

جدول (†): الإنفاق الاستثاري للمشروع

\begin{tabular}{|c|c|c|}
\hline القيمة المالية (مليون جنيه) & البيان & p \\
\hline 0. & الاجهزة شاملة الثحن والجمارك (المعجل) & 1 \\
\hline$\Lambda$ & الحواجز الخرسانية والمباني & T \\
\hline$r, 0$ & التجهيزات المكتبية والمعملية & $r$ \\
\hline $\bar{r}$ & وسائل النقل & $\varepsilon$ \\
\hline$r, 79$ & مصروفات ما قبل التشغيل & 0 \\
\hline$\cdot, 00$ & راس المال العامل (1) & 7 \\
\hline$\overline{V Y, \Sigma Y}$ & جمالى + احتياطى نقدي وعينى (· (\%) & \\
\hline
\end{tabular}

(1) مقدار شهر من إجمالى قيمة مصروفات النتغيل السنوية

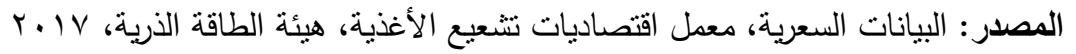
جدول (V): تكلفة العمالة السنوية

\begin{tabular}{|c|c|c|c|c|}
\hline الأجر السنوي للعاملين & 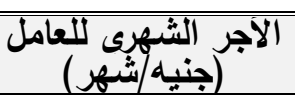 & العدد & البيان & s \\
\hline., 17 & $v \cdots$ & $\bar{r}$ & الإدارة العليا & 1 \\
\hline$\cdot, .9$ & YO.. & $r$ & الإدارة المتوسطة & $r$ \\
\hline$\cdot, 19 r$ & $\Lambda \ldots$ & r & العلميين & $r$ \\
\hline$\cdot, \mathrm{Y \Lambda}$ & $7 \ldots$ & $\varepsilon$ & الميندسين & $\varepsilon$ \\
\hline$\cdot, \mathrm{TV}$ & YO.. & 9 & فنيسن معامل لنتشغيل الوحدة & 0 \\
\hline$\cdot, 195$ & $r \ldots$ & $\Lambda$ & أمن وسائقين & 7 \\
\hline$\cdot, 1 \cdot 1$ & $10 \ldots$ & 7 & عمال شحن وتقفربغ & $\mathrm{V}$ \\
\hline $1, Y 0$ & & & لإ & \\
\hline
\end{tabular}

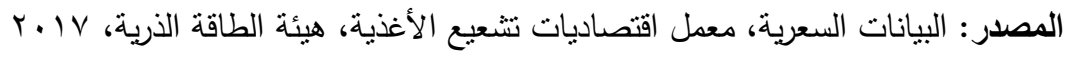
جدول (^): مصروفات التنغيل السنوية

\begin{tabular}{|c|c|c|}
\hline القيمة المالية (مليون جنيه) & البيان & p \\
\hline $1, Y 0$ & الأجور والرواتب & 1 \\
\hline$\cdot, 171$ & الكهرباء & $r$ \\
\hline $1, r V$ & الصيانة(1) & $r$ \\
\hline 1,10 & قطع غيار (ب) & $\varepsilon$ \\
\hline 1,0 & دعاية وترويج & 0 \\
\hline 7,01 & + احتياطى (·) & \\
\hline
\end{tabular}

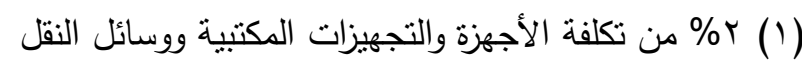

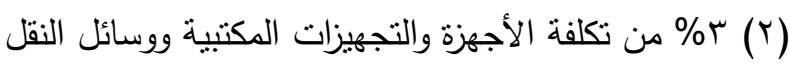

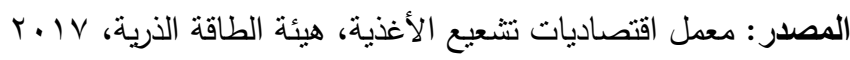


جدول (9): مصروفات ما قبل التشغيل

\begin{tabular}{|c|c|c|}
\hline القيمة المالية (مليون جنيه) & البيان & ? \\
\hline$\quad \cdot, 19$ & الاجور والرواتب (1) & $\frac{1}{1}$ \\
\hline $1, \cdots$ & دراسات وتصاريح & $\bar{r}$ \\
\hline 1,0 & دعاية وتزويج & 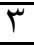 \\
\hline T,79 & الإجمالي & \\
\hline
\end{tabular}

$$
\text { (10 (10 من إجمالي تكاليف الأجور والمرتبات }
$$

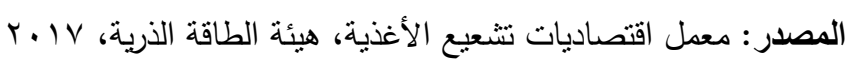

جدول ( • 1): القسط السنوى لإهلاك واستهلاك الأصول الرأسمالية

\begin{tabular}{|c|c|c|c|c|}
\hline 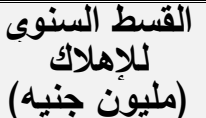 & (ملّيون جنيهة) & الصلاحية & البيان & b \\
\hline$\overline{T, 0}$ & 0. & $\overline{T .}$ & الاجهزة والمعدات & 1 \\
\hline$\cdot, \varepsilon$ & $\Lambda$ & $r$. & الحواجز الخرسانية والمبانِ & r \\
\hline$\cdot, 0$ & $r, 0$ & $\bar{V}$ & الاجهزة والمعدات الفنية والمكنيية & $r$ \\
\hline$\cdot, 19$ & T & $\mathrm{V}$ & وسائل النقل & $\varepsilon$ \\
\hline$\cdot, 1 T \leqslant$ & $r, 79$ & $r$ r. & مصروفات ما قبل التشغيلي(1) & 0 \\
\hline$r, \Lambda \mu$ & & & الإجم المِ & \\
\hline
\end{tabular}

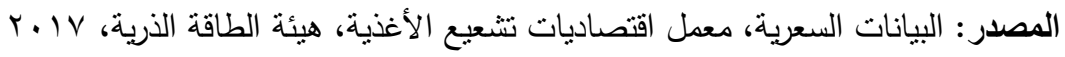

جدول (11): نكلفة التشغيل السنوية

\begin{tabular}{|c|c|c|}
\hline القيمة المالية (مليون جنيه) & البيان & م \\
\hline$T Y, 10$ & المرابحة على الإنفاق الاستثماري (1) & 1 \\
\hline 7,01 & مصروفات النتغيل السنوية & Tr \\
\hline$\Gamma, \Lambda \Gamma$ & القسط السنوي للإهلاك & $\Gamma$ \\
\hline rT,0T & الإجمالى & \\
\hline
\end{tabular}

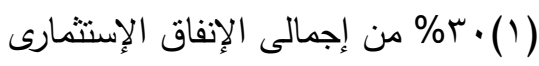

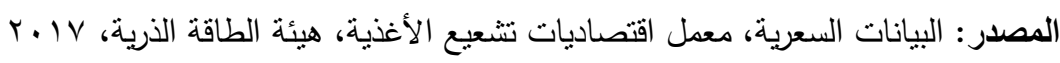

ثانياً: التدفقات الداخلة لوحدة المعجل الاكترونى بسوق العبور:

أ-النظام التسعيري والإيراد السنوى: تتحدد نسعيرة النتعيع وفقاً لـ (جنيه / ساعة نتشيل، جنيه

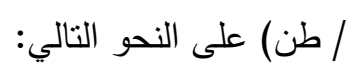


تسعيرة التشعيع (جنيه/ساعة تشغيل) = تكلفة التشغيل السنوية ؛ عدد ساعات التشغيل السنوية . . . . . . .

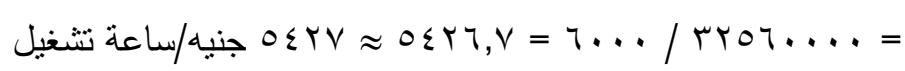
تسعيرة التشعيع (جنيه / طن) = تكلفة التشغيل السنوية ٪ الكمية المطلوب سنوياً تشعيعها

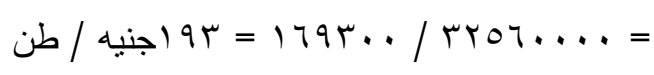

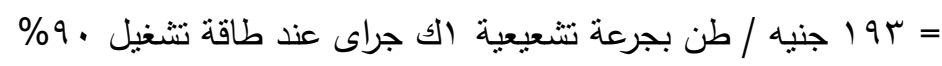

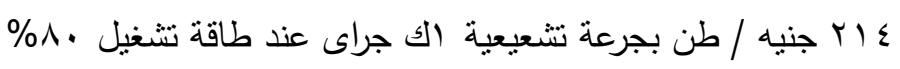

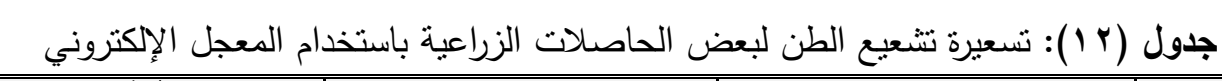

\begin{tabular}{|c|c|c|c|}
\hline سعنيه/التشعيع & الجرعة التشعيعية ك.جراى & المحصول & p \\
\hline TY &., 10 & البطاطس & 1 \\
\hline TY &., 10 & البصل & $r$ \\
\hline TY & $\cdot, 10$ & ألتوح & $r$ \\
\hline $11 Y$ & $\cdot, 0$ & البرتقال & $\varepsilon$ \\
\hline $7 \leqslant Y$ & $r$ & الفراولة & 0 \\
\hline $0 \mu, 0$ & $\cdot, Y_{0}$ & المانجو & 7 \\
\hline$T \backslash \varepsilon$ & $T$ & الرمان & V \\
\hline
\end{tabular}

سعر تثتعيع الطن =سعر تشعيع الطن بالجرعة الك.جراى × الجرعة التشعيعية الأصلية

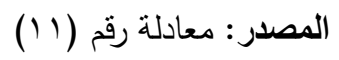
الإيراد السنوي: يتم حساب الإيراد السنوي وفقا للمعادلة الآتية: وذلك عند سعر تشعيع طاقة \%^ تشغيلية

الإيراد السنوي = الكمية المشععة سنوياً × سعر النتعيع معادلة رقم (r أ)

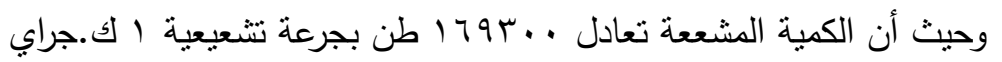

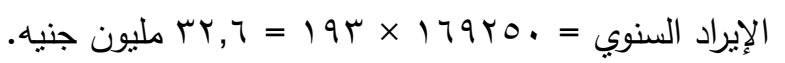

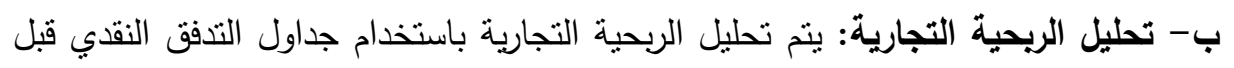
وبعد خصم الضرائب وتحليل الحساسية وذلك باستخدام معايير معدل العائد الداخلي للمشروع، فترة الاسترداد.

نتائج تقويم الريحية التجارية لإقامة وحدة تشعيع من نوع المعجل الإكتروني في سوق

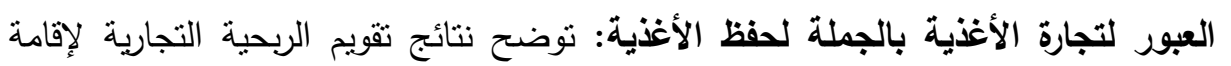

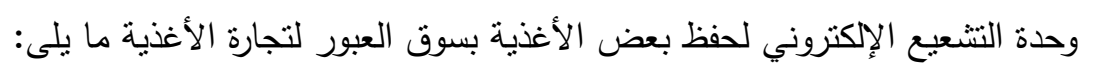


أ: في حالة التحليل الأساسي قبل فرض الضرائب:

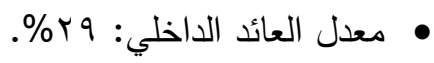

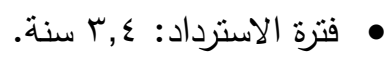

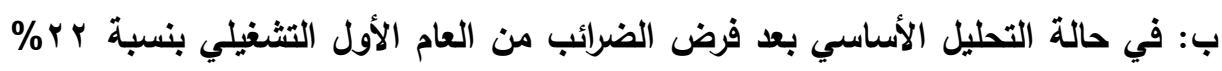
من إجمالي التدفق التقدي

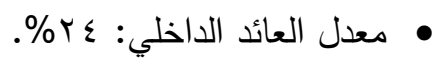
• • • فترة الاسترداد: r, ع سنة.

ت: في حالة تحليل الحساسية بافتراض زيادة مصروفات التشغيل وانخفاض الإيراد بنسبة \% أكل منهما.

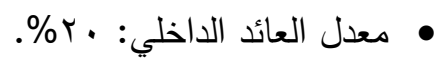

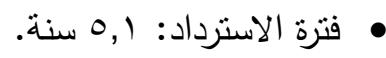

وبناء على ذلك فإن المشروع يعد مقبولاً وفقاً لنتائج تقويم الربحية التجارية من وجهة نظر

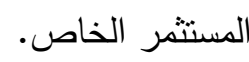


جدول (r ا (): حساب التدفقات النقدية للمشروع قبل خصم الضرائب (مليون جنيه)

\begin{tabular}{|c|c|c|c|c|c|c|c|}
\hline التضدي فئيل & السنْوي الإيراد & السنكالية & الألتجديا & التشنفوفيلي & الاستثماري & اللثظاقة & السنة \\
\hline $\bar{r} r, v-$ & - & $r \Psi, V$ & & - & $\overline{r y, V}$ & - & 1 \\
\hline$r q, V-$ & - & $r \overline{T, V}$ & & - & $r \bar{r}, \mathrm{~V}$ & - & $\bar{T}$ \\
\hline T1,vq & TO, ro & $r, 07$ & & $r, 07$ & & $\% \vee$. & $\Gamma$ \\
\hline$T 0, \varepsilon Y$ & $r \wedge, 9 \Lambda$ & $r, 07$ & & $\Gamma, 07$ & & $\% \wedge$. & $\varepsilon$ \\
\hline rq,,$\varepsilon$ & rY,T. & $r, 07$ & & $r, 07$ & & $\% 9$. & 0 \\
\hline$Y 7, \cdot Y$ & $r Y, T$. & 7,01 & & 7,01 & & $\% 9$. & 7 \\
\hline$r 7, \cdot T$ & $r, \tau$. & 7,01 & & 7,01 & & $\% 9$. & V \\
\hline$Y 7, \cdot Y$ & $r Y, T$. & 7,01 & & 7,01 & & $\% q$. & $\Lambda$ \\
\hline$Y 7, \cdot Y$ & TY,T. & 7,01 & & 7,01 & & $\% 9$. & 9 \\
\hline$Y \cdot, O Y$ & TY,T. & $T Y, \cdot \Lambda$ & 0,0 & 7,01 & & $\% 9$. & 1. \\
\hline$Y 7, \cdot Y$ & TY,T. & 7,01 & & 7,01 & & $\% 9$. & $\pi$ \\
\hline$Y 7, \cdot Y$ & TY,T. & 7,01 & & 7,01 & & $\% 9$. & TY \\
\hline$Y 7, \cdot Y$ & $r Y, T$. & 7,01 & & 7,01 & & $\% 9$. & $\pi$ \\
\hline$r 7, \cdot r$ & $r, \tau$. & 7,01 & & 7,01 & & $\% 9$. & $1 \varepsilon$ \\
\hline$Y 7, \cdot Y$ & $r Y, T$. & 7,01 & & 7,01 & & $\% 9$. & 10 \\
\hline$r 7, \cdot Y$ & TY,T. & 7,01 & & 7,01 & & $\% 9$. & 17 \\
\hline Y7,OV & $r \mu, 10$ & 7,01 & & 7,01 & & $\% 9$. & TV \\
\hline
\end{tabular}

(1) الثالاث سنوات التشغيلية الأولى بدون مصروفات صيانة وقطع غيار .

(Y) تم إضافة إسترداد رأس المال العامل فى الايراد السنوى للسنة الاخيرة.

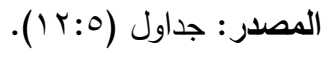


مجلة العلوم البيئية

معهد الدراسات والبحوث البيئية - جامعة عين شمس

حساب الضرائب على المشروع (مليون جنيه)

جدول (ـ 1): حساب الضرائب على المشروع (مليون جنيه)

\begin{tabular}{|c|c|c|c|c|c|c|c|}
\hline الضرائب & التضافي & السنتيوي الإيراد & الفي & 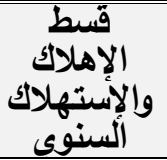 & التشغنيلي & 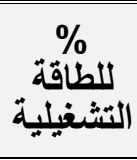 & السنة \\
\hline & & - & & & - & & $\overline{11}$ \\
\hline & & - & & & - & & $r$ \\
\hline r,90 & $1 V, 97$ & Yo, Mo & $v, r q$ & $\Gamma, \wedge \Gamma$ & r,oT & $\% \vee$. & r \\
\hline$\varepsilon, \vee 0$ & T1,09 & $r \wedge, q \wedge$ & $V, r q$ & $\Gamma, \wedge \mu$ & $r, 07$ & $\% \wedge$. & $\varepsilon$ \\
\hline 0,00 & $T 0, Y\}$ & r & $V, r q$ & $\Gamma, \Lambda \mu$ & $r, 07$ & $\% 9$. & 0 \\
\hline$\varepsilon, \wedge \wedge$ & TY,19 & Tr, I. & $1 \cdot, \leq 1$ & $\Gamma, \Lambda \mu$ & 7,01 & $\% 9$. & 7 \\
\hline$\varepsilon, \wedge \wedge$ & Tr,19 & Tr, I. & $1 \cdot, \leqslant 1$ & $\Gamma, \wedge r$ & $7,0 \wedge$ & $\% 9$. & $\mathrm{V}$ \\
\hline$\varepsilon, \wedge \wedge$ & Tr, Iq & rY, I. & $1 \cdot, \leqslant 1$ & $\Gamma, \Lambda \mu$ & 7,01 & $\% 9$. & $\Lambda$ \\
\hline$\varepsilon, \wedge \wedge$ & $r Y, 19$ & TY, T. & $1 \cdot, \leqslant 1$ & $\Gamma, \wedge r$ & 7,01 & $\% 9$. & 9 \\
\hline$\varepsilon, \wedge \wedge$ & $r Y, 19$ & Tr, I. & $1 \cdot, \leqslant 1$ & $\Gamma, \wedge \mu$ & 7,01 & $\% 9$. & $1 \cdot$ \\
\hline$\varepsilon, \wedge \wedge$ & $r Y, 19$ & Tr, I. & $1 \cdot, \leqslant 1$ & $\Gamma, \wedge \mu$ & 7,01 & $\% 9$. & 11 \\
\hline$\varepsilon, \wedge \wedge$ & Tr,19 & TY,I. & $1 \cdot, \leq 1$ & $\Gamma, \Lambda \mu$ & 7,01 & $\% 9$. & Tr \\
\hline$\varepsilon, \wedge \wedge$ & $r Y, 19$ & Tr, I. & $1 \cdot, \leq 1$ & T,AM & 7,01 & $\% 9$. & $\pi$ \\
\hline$\varepsilon, \wedge \wedge$ & $r Y, 19$ & $r$ r, & $1 \cdot, \leq 1$ & $\Gamma, \Lambda \mu$ & 7,01 & $\% 9$. & $1 \varepsilon$ \\
\hline$\xi, \wedge \wedge$ & $r Y, 19$ & TY, I. & $\mid \cdot, \leqslant 1$ & r,AM & 7,01 & $\% 9$. & 10 \\
\hline$\varepsilon, \wedge \wedge$ & TY,19 & Tr, & $1 \cdot, \leq 1$ & $\Gamma, \wedge \mu$ & 7,01 & $\% 9$. & 17 \\
\hline $0, \ldots$ & YY,VE & r & $1 \cdot, \leq 1$ & $\Gamma, \wedge \mu$ & $7,0 \wedge$ & $\% 9$. & $1 \mathrm{~V}$ \\
\hline
\end{tabular}

(1) الضرائب r \% من صافى التدفق النقدى فى السنة التشغيلية الأولى وهي السنة الثالثة

$$
\text { من عمر المشروع. }
$$

المصدر : مصلحة الضرائب المصرية، جداول (:0 (1). 
جدول (0 1): صافى التدفق النقدى للمشروع بعد خصم الضرائب

\begin{tabular}{|c|c|c|c|c|}
\hline صافى التدقّى التقدّي & الضرائب & 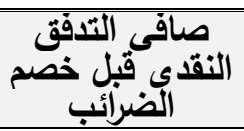 & 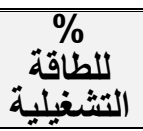 & السنة \\
\hline "r,v- & $\overline{-}$ & rq,V - & - & 1 \\
\hline$r \eta, V-$ & - & $r q, V-$ & - & $T$ \\
\hline$I V, \wedge \varepsilon$ & r,90 & FI, 19 & $\% \vee$. & $\Gamma$ \\
\hline$T \cdot, T V$ & $\varepsilon, \vee 0$ & YO, $\leqslant Y$ & $\% \wedge$. & $\varepsilon$ \\
\hline Tr, ₹q & 0,00 & $r q, \cdot \varepsilon$ & $\% 9$. & 0 \\
\hline$Y 1,1 \varepsilon$ & $\xi, \wedge \wedge$ & $Y 7, \cdot r$ & $\% 9$. & 7 \\
\hline$Y 1,1 \leq$ & $\varepsilon, \wedge \wedge$ & $Y 7, \cdot r$ & $\% 9$. & $\mathrm{V}$ \\
\hline$Y 1,1 \leq$ & $\varepsilon, \wedge \wedge$ & $Y Z, \cdot Y$ & $\% 9$. & $\Lambda$ \\
\hline$Y 1,1 \leq$ & $\varepsilon, \wedge \wedge$ & $Y 7, \cdot Y$ & $\% 9$. & 9 \\
\hline$Y 1,1 \leq$ & $\varepsilon, \wedge \wedge$ & $r q, \cdot Y$ & $\% 9$. & 1. \\
\hline $10,7 \varepsilon$ & $\varepsilon, \wedge \wedge$ & $Y Z, \cdot Y$ & $\% 9$. & 11 \\
\hline$Y, 1 \leq$ & $\varepsilon, \wedge \wedge$ & $Y 7, \cdot Y$ & $\% 9$. & Tr \\
\hline$Y 1,1 \leqslant$ & $\varepsilon, \wedge \wedge$ & $Y 7, \cdot Y$ & $\% 9$. & $\pi$ \\
\hline$Y 1,1 \leq$ & $\varepsilon, \wedge \wedge$ & $Y 7, \cdot r$ & $\% 9$. & $1 \varepsilon$ \\
\hline$Y 1,1 \leq$ & $\varepsilon, \wedge \wedge$ & $Y 7, \cdot r$ & $\% 9$. & 10 \\
\hline$Y 1,1 \leq$ & $\varepsilon, \wedge \wedge$ & $Y 7, \cdot r$ & $\% 9$. & 17 \\
\hline YI,OV & $0, \ldots$ & Y7,OV & $\% 9$. & IV \\
\hline
\end{tabular}

المصدر: جداول 0- ع 
جدول († 1): تحليل الحساسية للمشروع بافتراض زيادة مصروفات التشغيل وانخفاض الإيراد

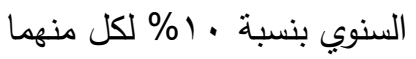

\begin{tabular}{|c|c|c|c|c|c|c|}
\hline صافِي التدقّق & بانَفَاضاضر & أنتكاليفب & الضرائب & مصروفات أنتشيادة & التشغيلية & السنة \\
\hline$\Gamma^{4}, \mathrm{~V}$ & - & $\overline{r q, V}$ & $=$ & $\overline{r q, V}$ & - & $T$ \\
\hline$r \tau, V-$ & - & $r q, V$ & - & $r q, V$ & - & $\bar{T}$ \\
\hline $1 \varepsilon, 90$ & $r Y, \wedge r$ & $\overline{V, \wedge V}$ & $r, 90$ & $r, q r$ & $\% \vee$. & $\bar{r}$ \\
\hline $\mid V, \Sigma)$ & $r \tau, \cdot \Lambda$ & $\Lambda, 7 V$ & $\varepsilon, V_{0}$ & $r, 9 Y$ & $\% \Lambda$. & $\varepsilon$ \\
\hline $19, \wedge V$ & $r q, r \varepsilon$ & $q, \leqslant V$ & 0,00 & $r, 9 r$ & $\% 9$. & 0 \\
\hline$T V, Y Y$ & Yq, r & $I T, I T$ & $\varepsilon, \wedge \wedge$ & $V, Y \leqslant$ & $\% 9$. & 7 \\
\hline$I V, Y Y$ & $r q, r \leqslant$ & $I T, I Y$ & $\varepsilon, \wedge \wedge$ & $V, Y \leqslant$ & $\% 9$. & V \\
\hline$I V, Y Y$ & $r q, r \varepsilon$ & $I T, I Y$ & $\varepsilon, \wedge \wedge$ & $V, Y \leq$ & $\% 9$. & $\Lambda$ \\
\hline$I V, Y Y$ & $r q, r \varepsilon$ & $I Y, I Y$ & $\varepsilon, \wedge \wedge$ & $V, Y \leqslant$ & $\% 9$. & 9 \\
\hline$T V, Y Y$ & $r q, r \varepsilon$ & $I V, T Y$ & $\xi, \wedge \wedge$ & $T Y, V \varepsilon$ & $\% q$. & 1. \\
\hline$T V, Y Y$ & $r q, r \varepsilon$ & $I Y, I Y$ & $\varepsilon, \wedge \wedge$ & $V, Y \varepsilon$ & $\% 9$. & 11 \\
\hline$I V, Y Y$ & $r q, \Gamma \varepsilon$ & $T, I Y$ & $\varepsilon, \wedge \wedge$ & $V, Y \varepsilon$ & $\% 9$. & $\pi$ \\
\hline$T V, Y Y$ & $r q, r \varepsilon$ & $T, I Y$ & $\varepsilon, \wedge \wedge$ & $V, Y \varepsilon$ & $\% 9$. & $\pi$ \\
\hline$T V, Y Y$ & rq, r $\varepsilon$ & $I T, I T$ & $\varepsilon, \wedge \wedge$ & $V, Y \leqslant$ & $\% 9$. & $1 \varepsilon$ \\
\hline$T V, Y Y$ & rq,r & $I T, I T$ & $\varepsilon, \wedge \wedge$ & $V, Y \leqslant$ & $\% 9$. & 10 \\
\hline$I V, Y Y$ & $r q, r \varepsilon$ & $I Y, I Y$ & $\varepsilon, \wedge \wedge$ & $V, Y \leq$ & $\% 9$. & 17 \\
\hline$\Pi 1, V Y$ & 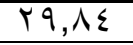 & $1 Y, 1 Y$ & $\varepsilon, \wedge \Lambda$ & $V, Y \leq$ & $\% 9$. & IV \\
\hline
\end{tabular}

العوائد القومية لمشروع إقامة وحدة تشعيع جامى بسوق العبور لتجارة الجملة:

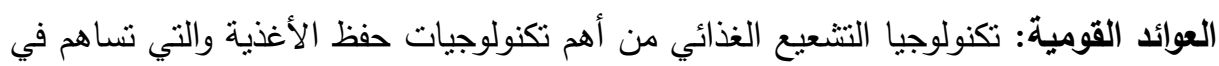

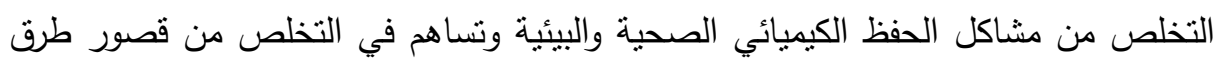
الحفظ الأخرى. ومن أهم العوائد القومية لحفظ الأغذية بالتثنعيع: الحفئ أولاً: تحسين الظروف البيئة. ثانياً: نحسين القدرات التسويقية للأغذية.

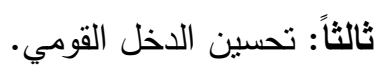


أولاً: دور تكنولوجيا المعالجة الأيونية للأغذية في تحسين الظروف البيئية:

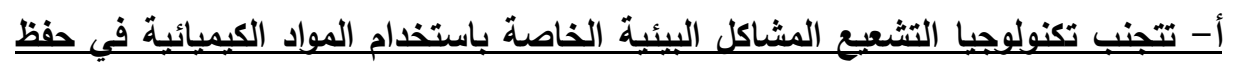
الأغذية وهي: • المشاكل البيئية والصحية الخاصة بالمتعاملين بالكيماويات. • المشاكل البيئية والصحية للبيئة المحيطة بالمعاملات الكيميائية.

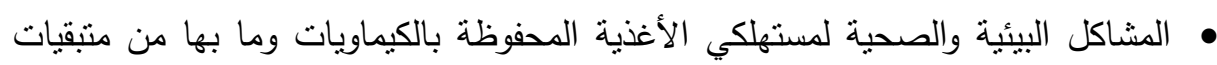

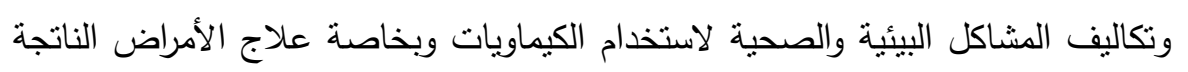
عنها وكذلك ضعف القدرات الانتاجية لأفراد المجتمع نتيجة الأمراض المتعلقة باستخدام الكيماويات.

\section{ب- تساهم تكنولوجيا التشعيع في خفض إستهلاك الطاقة الكهريائية} ثانياً: دور تكنولوجيا المعالجة الأيونية للأغذية في تحسين قدراتهائا التسويقية: تسهر

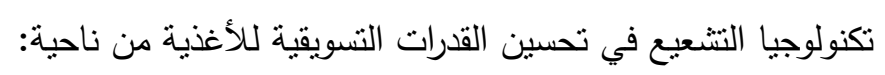
• خفض الفاقد الغذائي الكمي والنوعي. • • • • • إطالة فترة الحفظ بما يزيد من فترة تواجد السلعة الغذائية بالسوق.

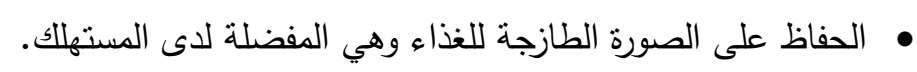

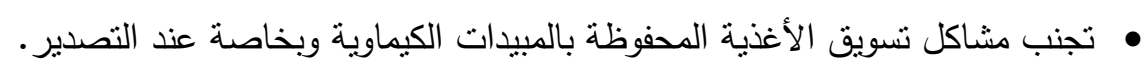
• الحفاظ على جودة وقيمة الغذاء. ثالثاً: دور تكنولوجيا المعالجة الأيونية للأغذية في زيادة الاخل القومي: أ- تساهم تكنولوجيالتشعيع في زيادة الدخل القومى من حيث:

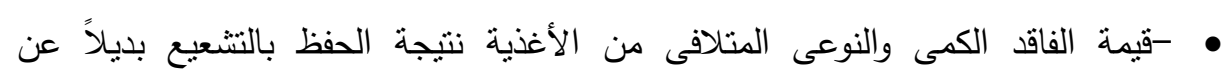
الكيماويات. • - قيمة تكاليف المشاكل الصحية والبيئية المتلافاة نتيجة الحفظ بالتشعيع بدلا من الحفظ

$$
\text { بالمبيدات الكيماوية. }
$$

ويوضح الجدول رقم (10) القيمة الدضافة إلى الدخل القومى عند إستخدام المعالجة

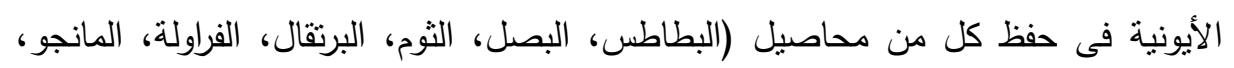


الرمان) حيث يوضح الجدول على سبيل المثال بالنسبة لمحصول البطاطس الكمية المقترح

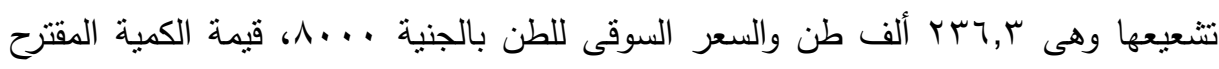

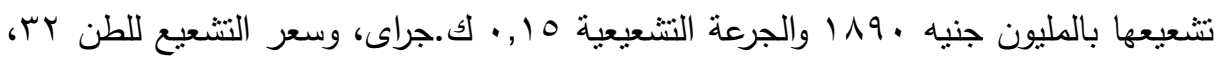
وتكاليف تشعيع الكمية الإجمالية V,OT مليون جنيه، وقيمة الفاقد المتلافى نتيجة التشعيع

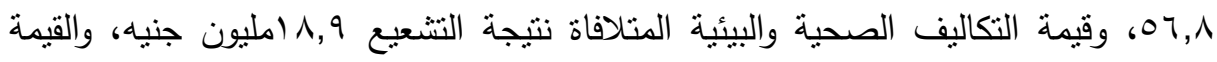

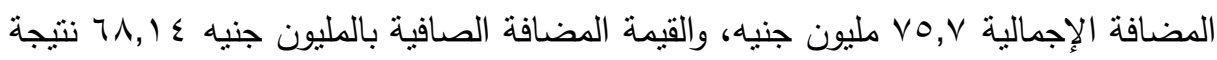
حفظ البطاطس بالتشعيع، وبالنسبة لإجمالى المحاصيل موضوع الدراسة فإن القيمة المضافة للاخل القومى عند إنشاء وحدة معجل إلكترونى بسوق العبور لحفظ كل من (البطاطس،

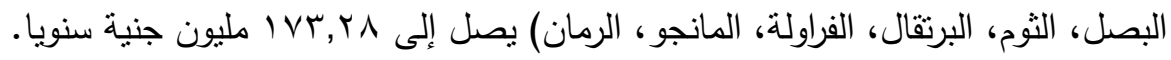

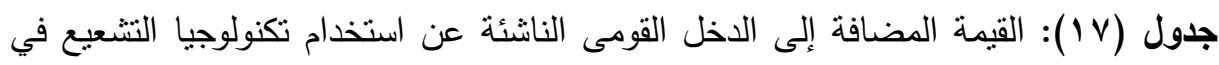
حفظ بعض الحاصلات الزراعية

\begin{tabular}{|c|c|c|c|c|c|c|c|c|c|c|}
\hline 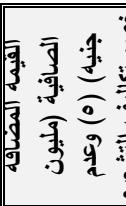 & 哥事 & $\overline{3}$ & 牙 & 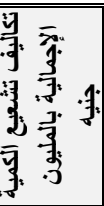 & $\begin{array}{l}3 \\
3.7 \\
3 \\
3 \\
3\end{array}$ & 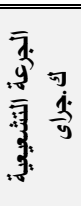 & 表 & 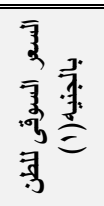 & 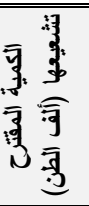 & $\overline{7}$ \\
\hline $7 \wedge, 1 \leqslant$ & $v_{0, v}$ & $1 \wedge, 9$ & 04,1 & $\mathrm{V,04}$ & Trr &., 10 & 119. & ${ }^{\prime \prime \ldots \ldots}$ & TrY, & البطاطس \\
\hline$r \varepsilon, r \varepsilon$ & $\varepsilon r, q r$ & $1,, \mathrm{VH}$ & rY,19 & $\Lambda, \diamond \Lambda$ & $r r$ &., 10 & $1 . v \mu$ & $\varepsilon \ldots$ & $r \Psi \Lambda, r$ & البصل \\
\hline $9,0 \mathrm{r}$ & 11,94 & $r, q \wedge$ & $\Lambda, q \leqslant$ & $r, r q$ & rr &., 10 & raA & $\varepsilon \ldots$ & $v \varepsilon, y$ & الثوم \\
\hline $11, v 1$ & $19,0 r$ & $\varepsilon, \wedge \wedge$ & $1 \leqslant, 1 \leqslant$ & $\mathrm{~V}, \wedge 1$ & $11 r$ &., 0. & $\varepsilon \wedge \wedge$ & $4 \ldots$ & $79, v$ & البرتقال \\
\hline$-1,1 \leqslant$ & $1, \wedge \wedge$ &.,$\leqslant \mathrm{V}$ & $1, \xi 1$ & $r, \cdot r$ & $T \leqslant Y$ & $r$ & $\varepsilon v$ & $1 \ldots$ & $\varepsilon, V$ & الفزاولة \\
\hline $0 ., 0 \mathrm{~V}$ & $00,0 Y$ & $1 T, \wedge \wedge$ & $\leqslant 1,1 \leqslant$ & $\varepsilon, 90$ & هr,o & . , ro & $1 T \wedge A$ & $10 \ldots$ & $9 Y, 0$ & المانجو \\
\hline . IV V & $r, \wedge \wedge$ &.,$V Y$ & $r, 19$ & $r, r)$ & ri\& & 1 & VY & $v \ldots$ & $1 \cdot, r$ & الرمان \\
\hline$I V \Psi, \Lambda Y$ & & & & & & & & & & الإجمالي \\
\hline
\end{tabular}

1- بيانات التسعيرة بسوق العبور لتجارة الأغذية بالجملة بمحافظة القاهرة.

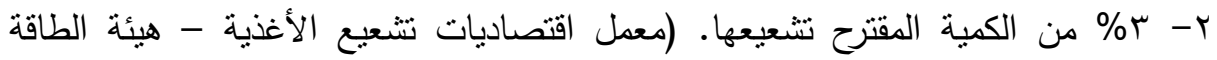
الذربة). r- 1\% من الكمية المقترح تشعيعها. (معمل اقتصاديات تشعيع الأغذية - هيئة الطاقة الذرية). 
ع - قيمة الفاقد المتلافي + قيمة التكاليف الصحية والبيئية المتلافاة.

0- القيمة المضافة الإجمالية - تكاليف التشعيع الإجمالية.

المصدر: جداول (17: (17)

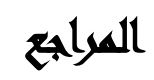

يوسف والي وآخرون (·9 (1)): حفظ الحاصلات البستانية وإعدادها وانضاجها وتخزينها وتصديرها، دار المعارف المصرية.

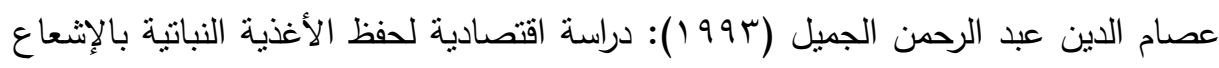

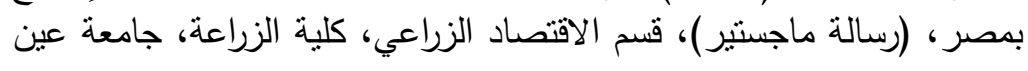
شمس.

محمد توفيق شرباص (1990): دراسة إمكانيات استخدام تكنولوجيا التشعع في حفظ وتخزين المنتجات الغذائية بالوطن العربي - جامعة الدول العربية للتنمية الزراعية.

إيمان محمد حسن (1991): دراسة جدوى لتكنولوجيا معالجة الأغذية بالإشعاع (رسالة

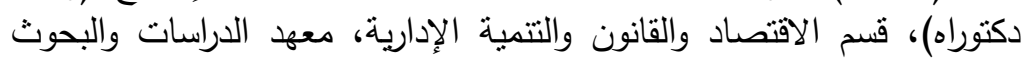

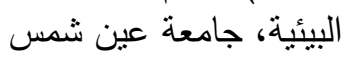

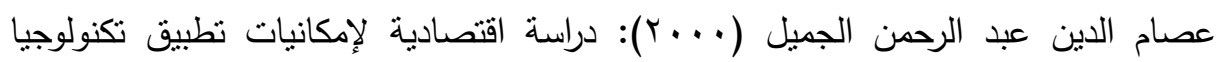

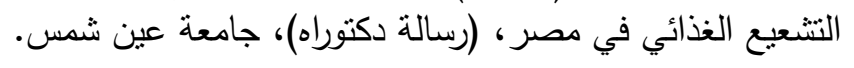

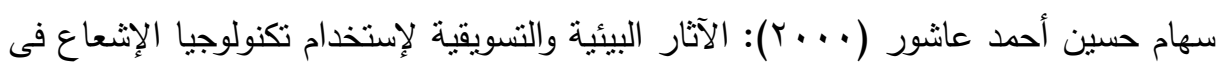

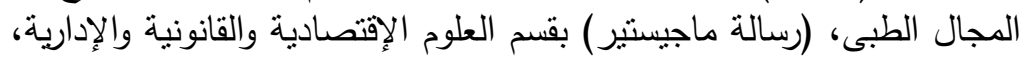

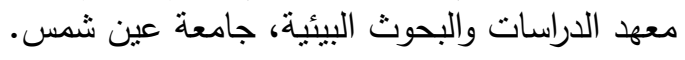

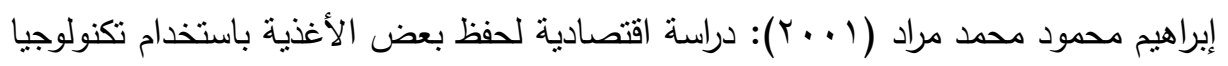

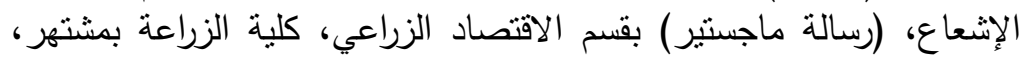
جامعة بنها.

عصام الدين عبد الرحمن الجميل: دراسة اقتصادية لإقامة وحدة لتتعيع الأغذية في ميناء بور الإمباء

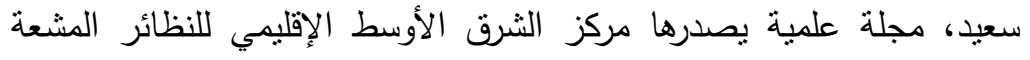

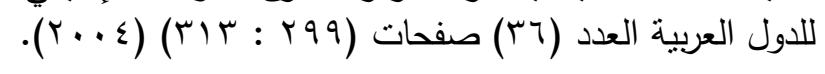




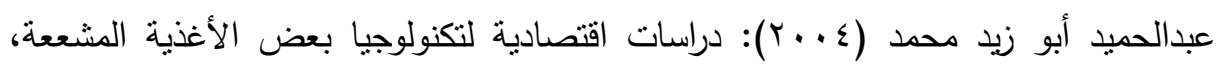

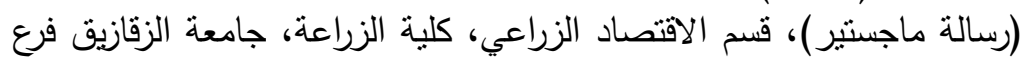

$$
\text { بنها. }
$$

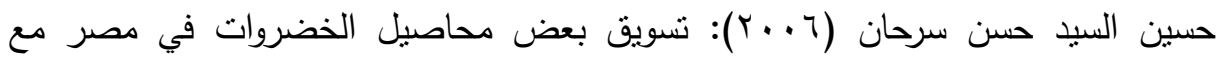

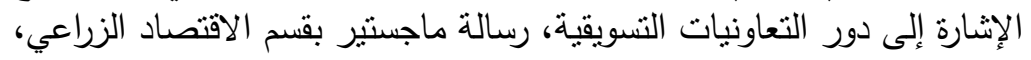
كلية الزراعة، جامعة عين شمس.

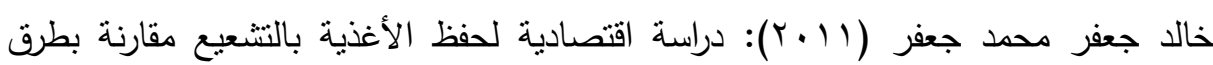

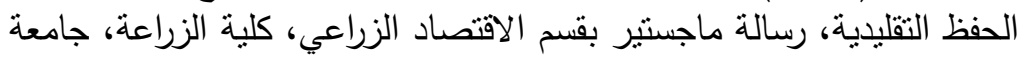

$$
\text { عين شمس. }
$$

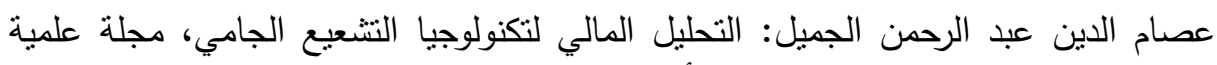

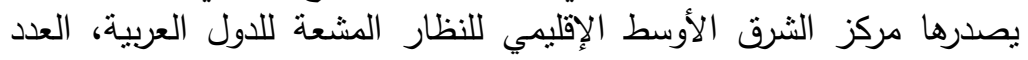

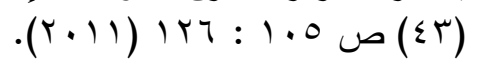

عصام الدين عبد الرحمن الجميل؛ مرفت عبد الرحمن الخطيب: دراسة اقتصادية لإقامة

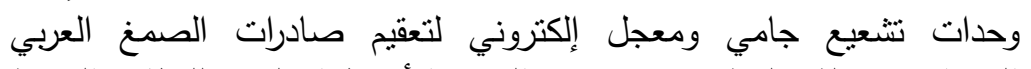

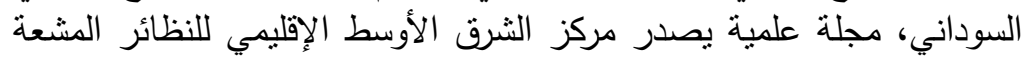

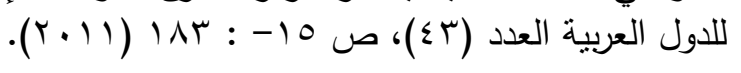

عصام عبد الرحمن الجميل؛ محمد مكاوي: دراسة اقتصادية لإقامة وحدة معجل إلكتروني

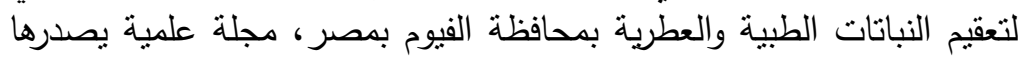

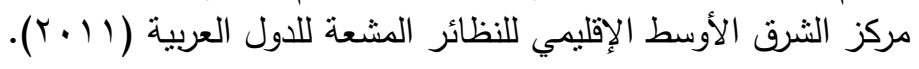

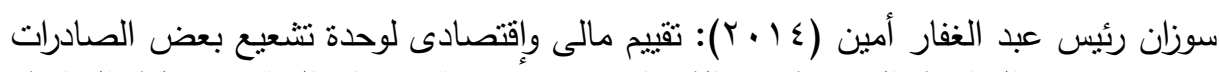

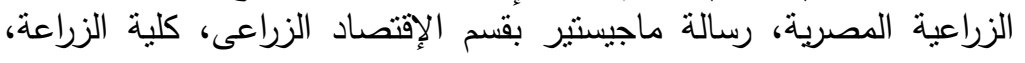

$$
\text { جامعة عين شمس. }
$$


علا صلاح عبد المجيد وآخرون

\title{
ENVIRONMENT ECONOMIC STUDY OF IONIC TREATMENT OF SOME AGRICULTURAL CROPS
}

\author{
Ola, S. Abdel Majeed ${ }^{(1)}$; Mohamed, S. Shehata ${ }^{(2)}$; \\ Essam, A. El-Gameel ${ }^{(1)}$ and Hisham, A. Al-Shura ${ }^{(2)}$
}

1) Atomic Energy Authority 2)Faculty of Agriculture, Ain Shams University

\begin{abstract}
Due to the environmental and health problems of the use of chemicals in food preservation since the preparation of this study to contribute to improving the environmental and health status of the community through an economic study to establish food irradiation unit in the El-oubowr market for food wholesale trade as an alternative to chemical use.

The study showed that potatoes, onions, garlic, orange, strawberries, mangoes and pomegranates The most important crops traded in the El-oubowr market, which need to be preserved by ionic treatment, where the study included the technical study to identify the appropriate doses for each crop through specialized studies in this area, To determine the future demand for the irradiation service during the lifetime of the project. The model of the appropriate irradiation units was then determined, by electronic accelerators. The financial analysis was carried out, including the determination of revenues and costs, as well as evaluating the commercial profitability and pricing system. The marketing capabilities of these agricultural crops to prolong the conservation period and preserve the value and quality of food and to avoid the health and environmental problems resulting from the use of chemicals. The study recommended the establishment of irradiation unit for these crops El-oubowr to prove its technical, marketing, financial and community feasibility.
\end{abstract}

Key words: food irradiation, losses, demand, financial analysis, social return.

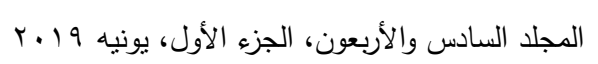

